\title{
syn-Selective Direct Asymmetric Cross-Aldol Reaction
}
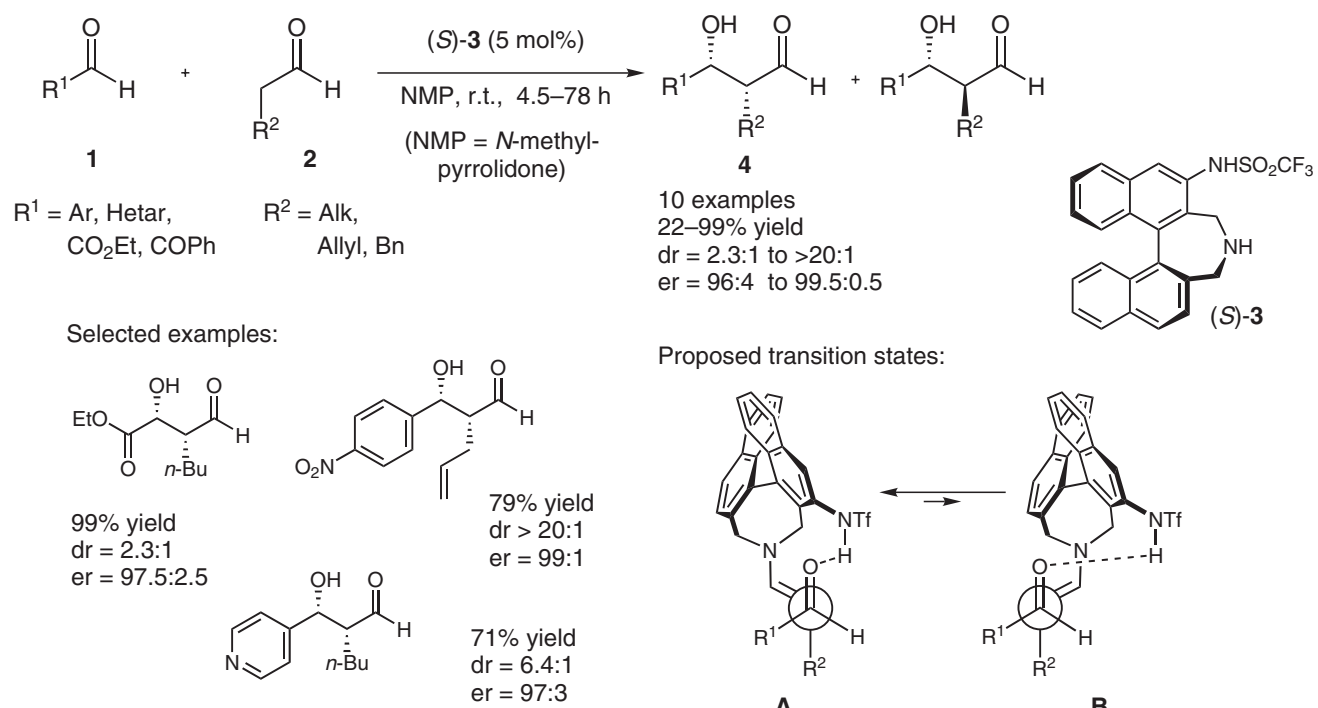

Proposed transition states:

Organo- and

Biocatalysis

Key words

cross-aldol reaction syn selectivity

axial chirality

amino sulfonamides

Significance: A highly syn-selective asymmetric direct cross-aldol reaction between two different aldehydes has been developed. By employing $5 \mathrm{~mol} \%$ of the axially chiral amino sulfonamide (S)-3, syn-aldol products 4 resulting from mostly electron-poor acceptor $\mathbf{1}$ and aliphatic donor aldehydes $\mathbf{2}$ are obtained in moderate to good yields along with high diastereo- (syn/anti ratio up to $>20: 1$ ) and excellent enantioselectivities (er up to $99.5: 0.5)$. The authors rationalize the stereochemical outcome with the help of proposed transition states $\mathbf{A}$ and $\mathbf{B}$. In contrast to the anti-enamine $\mathbf{B}$, the syn-enamine geometry in $\mathbf{A}$ allows for effective hydrogen bonding activation of the acceptor aldehyde by the acidic proton of the triflamide moiety. This favors the reaction to proceed via the syn-enamine intermediate and explains the observed syn selectivity.
Comment: The described method represents one of the rare examples of syn-selective direct crossaldol reaction proceeding via an enamine intermediate (e.g., C. F. Barbas, III and co-workers J. Am. Chem. Soc. 2007, 129, 288-289). With respect to the syn/anti selectivity, the use of axially chiral amino sulfonamide (S)-3 complements the proline-catalyzed version of both the direct asymmetric cross-aldol and the Mannich reaction, recently reported by the same group (J. Am. Chem. Soc. 2005, 127, 16408-16409). The drawback of multi-step catalyst synthesis is compensated not only by low catalyst loading but also by $95 \%$ catalyst recovery after column chromatography. A more general substrate scope with regard to the acceptor aldehyde might be desirable. 